\title{
AVOIDED CROSSINGS IN PHOTOCHEMISTRY
}

\author{
Alain Devaquet \\ Laboratoire de Chimie Théorique†, Centre Scientifique d'Orsay, \\ Université de Paris-Sud, 91405 ORSAY, France
}

\begin{abstract}
Two families of avoided crossings exist: (a) geometry-dependent, and (b) model-dependent. They are consequences of one of three possible molecular orbital patterns (allowed crossing, weakly avoided crossing and near-touching situations). All these cases are illustrated here and their mechanistic implications investigated both in terms of static potential energy surfaces and of dynamic Landau-Zener transition probabilities.
\end{abstract}

The non-crossing rule for potential energy curves of diatomic molecules was demonstrated fifty years ago ${ }^{1,2}$. Its extension to the general case of the potential energy surfaces of polyatomic molecules was only published very recently ${ }^{3,4}$. This long period of time might indicate that this fundamental rule, though invoked very frequently, has not yet received the full attention it deserves. Indeed, in organic photochemistry, its mechanistic implications have only been recognized during the past ten years. This was mainly due to the Woodward and Hoffmann pioneering study of forbidden thermal and allowed photochemical reactions ${ }^{5}$. On the other hand Van der Lugt and Oosterhoff were the first to emphasize the importance of avoided crossing regions as likely leakage channels from an excited state toward the ground state of reacting systems. Their calculations of the potential energy surfaces for the photochemically allowed closure of butadiene to cyclobutene beautifully illustrate this fact ${ }^{6}$.

Our goal in this paper is to review the various aspects of the avoided crossing problem. After a brief outline of its theoretical treatment we shall discuss this phenomenon in terms of electronic states and then, more precisely, at the molecular orbital level. We shall conclude with notes on the mechanistic consequences of this rule in various areas of photochemistry.

\section{TWO-STATE MODEL FOR AYOIDED CROSSINGS}

Let us suppose that the potential energy surfaces $d_{1}$ and $d_{2}$ associated with the states $\phi_{1}$ and $\phi_{2}$ of a given system cross for a value $r_{\mathrm{c}}$ of a group of nuclear coordinates. In rather loose terms the physical situation associated with this

$\dagger$ Also part of the Laboratoire de Physico-Chimie des Rayonnements associated with the CNRS. 


\section{ALAIN DEVAQUET}

crossing may be pictured in the following manner. Were these two states completely independent the system moving along $d_{1}\left(d_{2}\right)$ would have no knowledge of the existence of $d_{2}\left(d_{1}\right)$ and would pass through the crossing region without being perturbed. In this particular case $\phi_{1}\left(\phi_{2}\right)$ will remain everywhere an exact solution of the Schrödinger equation. If, on the contrary the wavefunctions $\phi_{1}$ and $\phi_{2}$ are not independent of each other and have some common properties the system at $r_{\mathrm{c}}$ will find itself in a very ambiguous situation. It may stay on $d_{1}$ or switch, more or less easily, to $d_{2}$. Half of the time the system will then be described by $\phi_{1}$, the other half by $\phi_{2} . \phi_{1}$ and $\phi_{2}$ are no longer exact solutions of the Schrödinger equation. They are called 'diabatic' wavefunctions. The exact-or adiabatic-solutions $\psi_{1}, \psi_{2}$ will be equal mixtures of these diabatic functions. The only point to be clarified is the nature of the common characteristics linking the two wavefunctions $\phi_{1}$ and $\phi_{2}$. The most general spatial property of two states, whatever their intimate electronic nature, is their symmetry. Therefore the physics of the problem at hand may be summarized as follows:

(1) if two states of different symmetry cross, they will ignore each other. Their wavefunctions remain unperturbed and are adiabatic solutions of the Schrödinger equation. The crossing is strictly allowed [Figure 1(a)].

(2) if they have the same symmetry, the two states will only be diabatic solutions of the problem. They will mix with each other to give two adiabatic solutions which no longer cross. The crossing becomes avoided [Figure $1(b)]$.

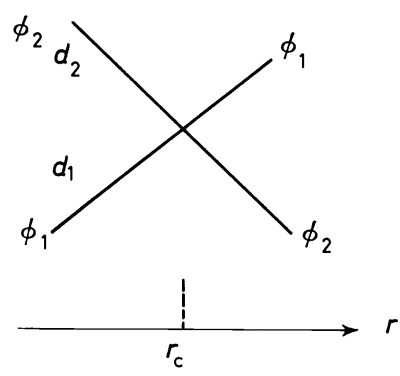

(a) Allowed crossing

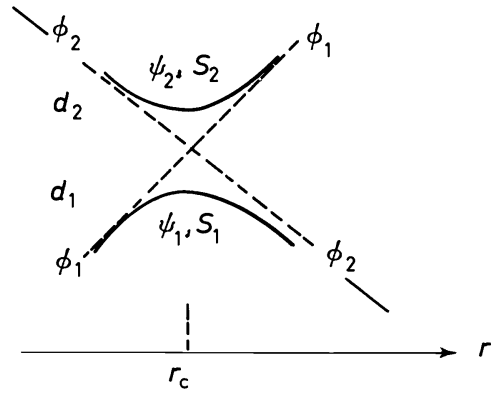

(b) Avoided crossing

Figure 1. General representation of (a) the allowed crossing between states of different symmetry, and (b) the avoided crossing between states of similar symmetry.

The mathematical formulation of the mixing problem is straightforward. The adiabatic solutions of the Schrödinger equation, $\psi_{1}$ and $\psi_{2}$, are searched as linear combinations of the diabatic ones

$$
\psi_{1,2}=C_{1} \phi_{1}+C_{2} \phi_{2}
$$

The variation method is used to solve for the coefficients ${ }^{7} C_{1}$ and $C_{2}$. In 


\section{AVOIDED CROSSINGS IN PHOTOCHEMISTRY}

the simplest case where the overlap between $\phi_{1}$ and $\phi_{2}$ may be neglected the

$$
\Delta=\left|\begin{array}{ll}
\varepsilon_{1}-E & H_{12} \\
H_{12} & \varepsilon_{2}-E
\end{array}\right|
$$

determinant $\Delta$ must vanish. In this determinant $\varepsilon_{1}$ and $\varepsilon_{2}$ are the energies of $\phi_{1}$ and $\phi_{2}$ at a given $r$ (at the crossing point $r_{\mathrm{c}}: \varepsilon_{1}=\varepsilon_{2}=\varepsilon_{\mathrm{c}}$ ) and $H_{12}$ is the matrix element of the electronic hamiltonian $H$ of the system between $\phi_{1}$ and $\phi_{2}$, i.e. $H_{12}$ is the term responsible for the mixing of $\phi_{1}$ and $\phi_{2}$. At $r_{\mathrm{c}}$ the two possible adiabatic wavefunctions and their respective energies are simply:

$$
\begin{gathered}
\psi_{2}^{1}=\left(\phi_{1} \pm \phi_{2}\right) / \sqrt{ } 2 \\
E_{2}^{1}=\varepsilon_{\mathrm{c}} \pm H_{12}
\end{gathered}
$$

At $r_{\mathrm{c}}$, the gap $g$ between the two adiabatic states is twice the coupling term $H_{12}$.

$$
g=2 H_{12}
$$

Of course, such a mixing is not a particular property of the point $r_{\mathrm{c}}$. Instead it is a continuous phenomenon. The diagonalization procedure may be repeated for each point $r$ in the vicinity of $r_{\mathrm{c}}$. At such points $\varepsilon_{1}$ and $\varepsilon_{2}$ are obviously slightly different and the weights of $\phi_{1}$ and $\phi_{2}$ become unequal. The magnitude of the mixing decreases when $r$ goes farther away from $r_{\mathrm{c}}$ or, in other words, when the coupling element $H_{12}$ becomes smaller compared to the energy gap $\varepsilon_{2}-\varepsilon_{1}$ between the diabatic functions. When $r$ is sufficiently different from $r_{c}$ the mixing is negligible and the adiabatic and diabatic solutions become identical. If then we consider the lowest state $\psi_{1}$, it is identical with $\phi_{1}$ when $r \ll r_{\mathrm{c}}$ but acquires more and more $\phi_{2}$ character when $r$ approaches $r_{\mathrm{c}}$. At $r_{\mathrm{c}}, \psi_{1}$ is an equal mixture of $\phi_{1}$ and $\phi_{2}$. When $r$ becomes greater than $r_{\mathrm{c}}$ the $\phi_{1}$ character gradually decreases. At $r \gg r_{\mathrm{c}}, \psi_{1}$ is identical with $\phi_{2} . \psi_{2}$ exhibits the reverse behaviour. A consequence of the mixing is to repel the two states so that $\psi_{1}$ and $\psi_{2}$ no longer cross. Let us finally note that when the two states $\phi_{1}$ and $\phi_{2}$ have different symmetry $H_{12}$ is identically zero. The two roots of the determinant $\Delta$ are simply $\varepsilon_{1}$ and $\varepsilon_{2}$ and the two adiabatic functions are still $\phi_{1}$ and $\phi_{2}$. There is no energy gap at $r_{\mathrm{c}}$ and the crossing is strictly allowed.

We know then that in the region of avoided crossing the two adiabatic curves approach each other very closely. It is likely that the system will be able to pass from one potential energy surface to the other. What is the probability for such a jump to occur? This dynamic (time-dependent) problem may be treated in a way which somewhat parallels the static treatment $^{8-11}$. If we notice that the probability of decay from one adiabatic surface $S_{2}$ to the other $S_{1}$ is the same as that for remaining on the diabatic (intersecting) surface $d_{2}$ it appears again convenient to express the $\psi \mathrm{s}$ in terms of the $\phi$ s. However, we have to consider the changes of the system as a function of the time $t$ instead of the nuclear coordinate $r$. If the transition region-the avoided crossing region-is sufficiently small the diabatic functions $\phi_{1}$ and $\phi_{2}$ may be considered as locally independent of the time. They do not, however, represent steady states since the system jumps from 


\section{ALAIN DEVAQUET}

$\phi_{1}$ to $\phi_{2}$ at the crossing. The mixing coefficients $C_{1}$ and $C_{2}$ must therefore be time-dependent. The trial wavefunction of the system is written

$$
\psi=C_{1}(t) \exp \left[\{2 \pi i / h\} \varepsilon_{1} t\right] \phi_{1}(r)+C_{2}(t) \exp \left[\{2 \pi i / h\} \varepsilon_{2} t\right] \phi_{2}(r)
$$

The second difference with the previous static treatment is that we must replace the time-independent electronic hamiltonian $H$ by the time-dependent operator, i.e.

$$
H-(h / 2 \pi i)(\partial / \partial t)=H(t)
$$

The two coupled differential equations which represent the variations of $C_{1}(t)$ and $C_{2}(t)$ are solved asymptotically and the probability $P$ of decay from one adiabatic surface to the other is given by an expression first obtained by Landau and Zener ${ }^{8,9}$

$$
P=\exp \left\{\left(-\pi^{2} / h\right)\left(g^{2} / V \delta s\right)\right\}
$$

This transition probability will be close to unity when the quantity $g^{2} / V \delta s$ is small. Let us then comment briefly on the three factors involved in this expression. $g$ is the energy gap between the two adiabatic states at the crossing point. It is obvious that the smaller this gap is, the greater will be the probability of a 'jump'. $\delta s$ is the difference of the slopes of the two crossing diabatic surfaces at $r_{\mathrm{c}}$. It may be intuitively understood how the transition probability increases with $\delta s$ (Figure 2). In situation (a) the nature of, say, $\psi_{2}$ changes

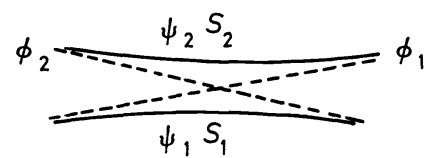

(a) $\delta S$ small

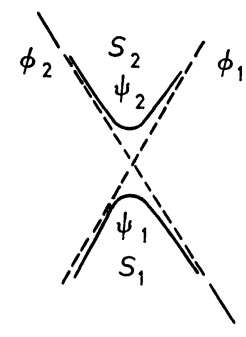

(b) $\delta S$ large

Figure 2. Small (a) and large (b) difference of the slopes of the diabatic (intersecting) potential energy surfaces in the case of an avoided crossing.

smoothly in the crossing region. The system adapts itself to this modification quite easily and has no reason not to remain on $S_{2}$. On the contrary the situation (b) is characterized by a sudden change of both the nature of $\psi_{2}$ and the slope of $S_{2}$ in the vicinity of $r_{\mathrm{c}}$. The system cannot cope with these drastic variations and, to avoid them, decays on to the surface below. These two first factors, $g$ and $\delta s$, may be obtained from quantum-mechanical calculations - $a b$ initio, for example - of the two potential energy surfaces. This is not so with the remaining third factor $V$, the velocity with which the system crosses the avoided region. The greater this velocity, the greater the 


\section{AVOIDED CROSSINGS IN PHOTOCHEMISTRY}

probability of the jump since for large kinetic energies the system is carried on through $r_{\mathrm{c}}$ as though it were ignoring the avoided nature of the crossing.

\section{GEOMETRY AND MODEL-DEPENDENT AVOIDED CROSSINGS}

Avoided crossings may be divided into two large families. The first type (geometry-dependent avoided crossings) occurs in the near vicinity of a strictly allowed crossing between electronic states of different symmetry. If a small distortion of the nuclear skeleton destroys the symmetry element which differentiates the states, the crossing will become avoided. The second type (model-dependent avoided crossings) occurs when two crossing states are represented by crude wavefunctions which are not allowed to mix together. When this restriction is lifted, the mixing of the zeroth-order functions gives more accurate wavefunctions which no longer cross. Let us then investigate these two families:

\section{(1) Geometry-dependent avoided crossings}

Such avoided crossings occur very frequently. A simple example is the case of systems with only one symmetry element: a plane. In such a situation symmetric $\left(\mathrm{A}^{\prime}\right)$ and antisymmetric $\left(\mathrm{A}^{\prime \prime}\right)$ states will cross. If one or several nuclei are displaced slightly so as to destroy the symmetry plane all states will have the same symmetry (no symmetry at all) and all the crossings will become avoided. Examples of such situations in organic photochemistry have recently been discussed by L. Salem ${ }^{12}$, among them $(\sigma \pi)$ bitopic reactions (such as hydrogen abstraction by ketones) and certain $\sigma(\sigma \pi)$ tritopic reactions (such as the $\alpha$-cleavage of hexadienones). As an illustration let us briefly consider the typical reaction where formaldehyde abstracts a hydrogen from methane. On the left of Figure 3 the formaldehyde plane is a symmetry element for the whole supermolecule $\left(\mathrm{H}_{2} \mathrm{CO} \cdots \mathrm{CH}_{4}\right)$. The symmetric $\left(\mathrm{A}^{\prime}\right)$ ground state (which correlates with a high-energy zwitterionic intermediate) strictly crosses the antisymmetric $\left(\mathrm{A}^{\prime \prime}\right) \mathrm{n} \pi^{*}$ singlet state (which correlates with a relatively low-lying diradical). If the symmetry plane is suppressed by pyramidalizing the formaldehyde molecule these two states will mix. As a result the crossing becomes avoided (Figure 3, right). The singlet ground state now correlates with the diradical form of the intermediates but can still be recognized as of pseudo- $\mathrm{A}^{\prime}$ symmetry before and pseudo- $\mathrm{A}^{\prime \prime}$ symmetry after the avoided crossing. The maximum in the potential energy surface of the ground state and the secondary minimum in the excited state surface show up as a 'memory' of the intended crossing. This mixing region is one of sudden state-symmetry interchange. In a one-electron description of the physical process we would say that one electron suddenly switches from a $\sigma$ to a $\pi$ molecular orbital (or conversely). In the ground state of our example one electron is taken out of the $\mathrm{O}-\mathrm{H}-\mathrm{C} \sigma$ region into the carbonyl $\pi$ region.

\section{(2) Model-dependent avoided crossings}

We shall now investigate in both the valence bond (VB) and molecular orbital (MO) approaches the cases where an avoided crossing results from the improvement of crossing zeroth-order wavefunctions. 


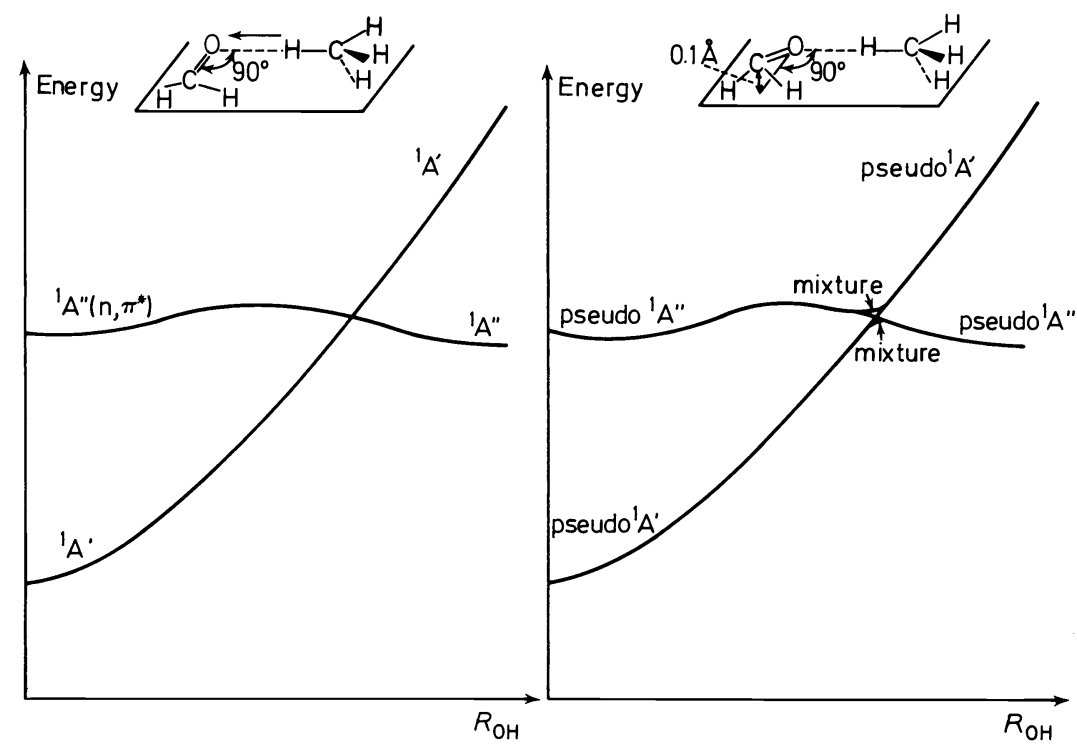

Figure 3. Energy surfaces for the formaldehyde-plus-methane hydrogen abstraction reaction: (a) a plane of symmetry exists; (b) the plane of symmetry has been destroyed.

The best known type of VB avoided crossing is probably the case of sodium chloride ${ }^{13,14}$. This molecule is essentially $\mathrm{Cl}^{-} \mathrm{Na}^{+}$near its equilibrium bond distance $(2.36 \AA)$ and yet dissociates to form atoms ${ }^{15,16}$. The zeroth-order 'purely ionic' ground state wavefunction

$$
\psi_{\mathrm{ION}}=\phi_{\mathrm{Cl}}(1) \phi_{\mathrm{Cl}}(2)
$$

correlates at infinity with the ion-pair $\mathrm{Cl}^{-} \mathrm{Na}^{+}$. Its potential energy curve must therefore cross that for the 'purely covalent' excited state

$$
\psi_{\mathrm{COV}}=\phi_{\mathrm{Cl}}(1) \phi_{\mathrm{Na}}(2)+\phi_{\mathrm{Cl}}(2) \phi_{\mathrm{Na}}(1)
$$

which dissociates into the radical pair $\mathrm{Cl}^{\circ}+\mathrm{Na}^{\circ}$. The allowed intersection of the two curves is a direct consequence of the unsophisticated character of the theoretical model. If we lift the condition of 'purity', $\psi_{\mathrm{ION}}$ and $\psi_{\mathrm{COV}}$ will mix in the crossing region to provide us with a more accurate representation of the real states of the molecule. As a natural consequence the crossing is weakly avoided. The gap $g$ between the adiabatic states (in the case where the overlap between $\psi_{\text {ION }}$ and $\psi_{\mathrm{COV}}$ is neglected) will be twice the matrix element $\left\langle\psi_{\text {COV }}|H| \psi_{\text {ION }}\right\rangle$ where $H$ denotes the total hamiltonian of the molecule. Both one-electron and two-electron terms in $H$ will give contributions. The ground state of the molecule, ionic at equilibrium, will correlate with the covalent diradical after going through a region of mixed character (Figure 4). Physically this corresponds to the 'jump' of one electron from the chlorine to the sodium atom. The internuclear distance at which this jump occurs, i.e. the zeroth-order states crossing distance $r_{\mathrm{c}}$, is quite large $(9 \AA$ for 


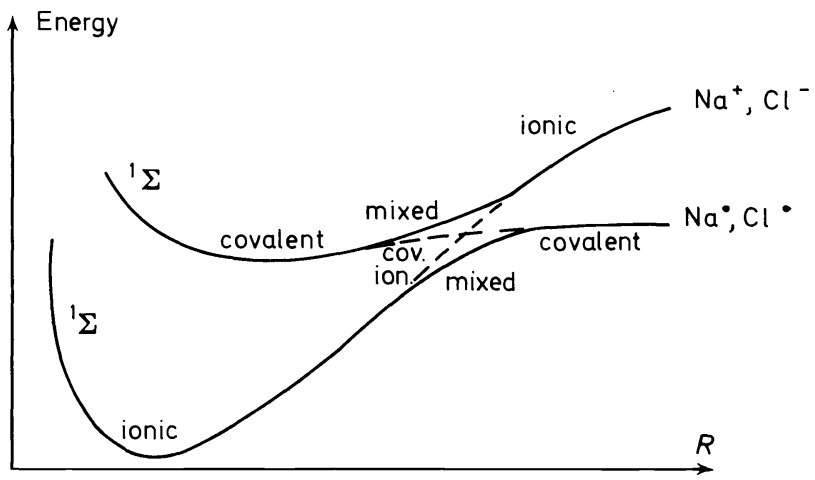

Figure 4. Avoided crossing between the ionic and covalent potential energy curves in the dissociation of sodium chloride.

$\mathrm{NaCl}$ ) and, since the Coulombic attraction is dominant there, may be easily determined from the energy required to form the ion-pair:

$$
e^{2} / r_{\mathrm{c}} \simeq I(\mathrm{Na})-E(\mathrm{Cl})
$$

Here $I(\mathrm{Na})$ denotes the ionization potential of the alkali atom and $E(\mathrm{Cl})$ the electron affinity of the halogen atom.

Crossings allowed at a crude level and becoming avoided at a higher level of description are also quite frequent in MO theory. In the MO methods electrons are moving independently of each other. They are ascribed to specific one-electron molecular orbitals which are the eigenfunctions of a monoelectronic operator, say, $F$. $F$ includes exactly the monoelectronic terms (kinetic energy and nuclear-electron attraction energy) but only an average of the bielectronic terms (electron-electron repulsion). $F$ is therefore slightly different from the real instantaneous (mono and bielectronic) hamiltonian $H$ of the system. At this level the symmetry allowed crossing which may occur between the highest occupied MO (HOMO) and the lowest unoccupied MO (LUMO) along some reaction coordinate is carried over in the potential energy surfaces for the corresponding one-electron configurations. In the photochemical disrotatory closure of butadiene into cyclobutene the HOMO ( $\pi_{2}$, antisymmetric) of butadiene correlates with the $\pi^{*}$ antibonding MO of cyclobutene and must cross the LUMO $\left(\pi_{3}\right.$, symmetric) of butadiene which correlates with the $\pi$ bonding $\mathrm{MO}$ of cyclobutene ${ }^{17,18}$. The surfaces for the configurations $\mathrm{S}^{2} \mathrm{~A}^{2}$ (ground state of butadiene) and $\mathrm{S}^{2} \mathrm{~S}^{2}$ (doubly excited state of butadiene) also cross [Figure 5 (a) and (b)]. This is due to the fact that the two configurations differ by the location of two electrons (both respectively in A and S) and cannot be mixed by the oneelectron operator $F$. However, the description of the states as one-electron configurations is incorrect in the region where these configurations have nearly equal energies ${ }^{19}$. The inclusion of the bielectronic repulsion terms in the form of the instantaneous repulsion (which is the bielectronic part of $H$ ) minus the average repulsion (which is only considered in $F$ ) will mix the two configurations ${ }^{20}$. The crossing which was allowed at the one-electron level 


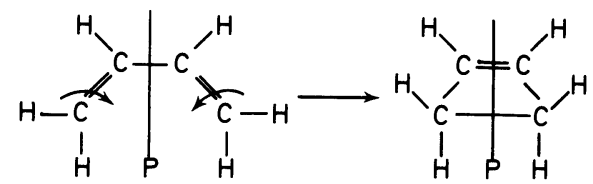

(a) orbitals

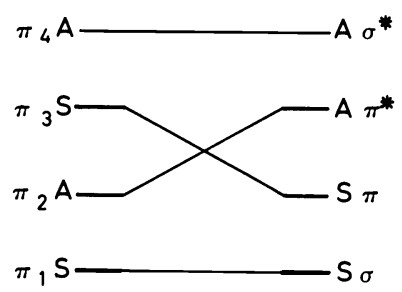

(b) configurations and states

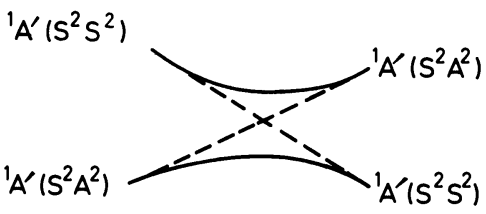

Figure 5. Disrotatory closure of butadiene in cyclobutene.

becomes forbidden at this second stage where the electron correlation is included [Figure 5 (b)]. The gap $2 \mathrm{H}_{12}$ between the two adiabatic states will be twice the exchange integral $K_{\mathrm{SA}}$ between the intersecting molecular orbitals. This exchange integral is usually small unless $\mathbf{S}$ and $\mathrm{A}$ are located over the same set of atoms, as in antiaromatic transition states. This is the case of the disrotatory closure of butadiene, to which we will return later on, and the coplanar (supra-supra) decomposition of cyclobutane into two ethylenes where the exchange integral is roughly $2.5 \mathrm{eV}^{21}$. The essential characteristics of the avoided crossing region is a switch in the major configuration which composes either state. Loosely speaking there is here a 'double electronic jump'. In the case of the ground state of our example two electrons leave simultaneously the A orbital to end up in the S orbital.

\section{MOLECULAR ORBITAL NATURE OF AVOIDED CROSSINGS}

The highest occupied molecular orbitals play a major role in the occurrence of avoided crossings between electronic states. This is not surprising. On the one hand they contain the valence electrons, and, as such, are easily perturbed during a reaction. On the other hand if a bonding level intends to correlate with an antibonding level a likely candidate to cross the corresponding energy gap will usually be the highest occupied MO.

(1) The most common MO pattern producing an avoided crossing of electronic states is the allowed crossing of two MOs. We have already encountered one such example. In the disrotatory closure of butadiene the antisymmetric $\pi_{2}$ MO of butadiene intersects the symmetric $\pi_{3}$ MO. These MOs have different symmetry and do cross. The ground state and the doubly excited $\left(\pi_{2} \rightarrow \pi_{3}\right)^{2}$ state of butadiene are both symmetric and their potential energy surfaces avoid each other. (A similar situation occurs in the suprasupra addition of two ethylenes to form cyclobutane ${ }^{5}$.) An example of avoided 


\section{AVOIDED CROSSINGS IN PHOTOCHEMISTRY}

crossing between singly excited states may be found in the conrotatory closure of butadiene ${ }^{17}$. Here two MOs allowed crossings are involved, the first one between the butadiene $S$ and $A$ occupied orbitals, the second between the butadiene $\mathrm{S}$ and A vacant orbitals [Figure 6(a)]. As a consequence the two antisymmetric singly excited states $\left(\pi_{2} \rightarrow \pi_{3}\right)$ and $\left(\pi_{1} \rightarrow \pi_{4}\right)$ which cross

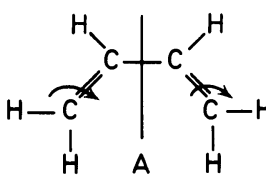

(a) orbitals

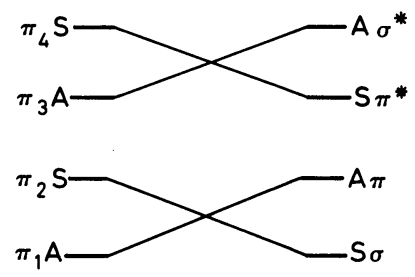

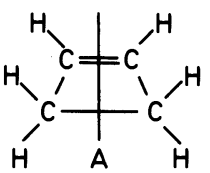

(b) configurations and states

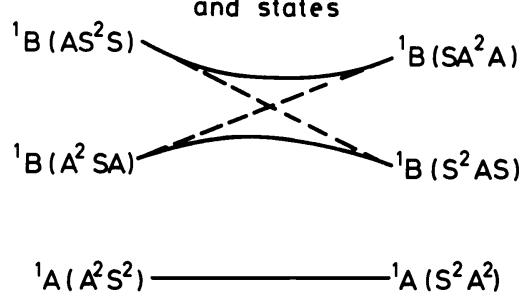

Figure 6. Conrotatory closure of butadiene in cyclobutene.

in the one-electron configuration approximation repel each other when the electron correlation is taken into account [Figure 6(b)]. In the crossing region two electrons simultaneously jump from one pair of orbitals $\left(\pi_{2}, \pi_{3}\right.$ of butadiene for example) to another pair ( $\pi, \pi^{*}$ of cyclobutene).

(2) A 'weakly avoided crossing' of MOs may also be thought of as being responsible for states avoided crossings. A beautiful example of this is the coplanar dissociation ${ }^{22}$ of ammonia to $\mathrm{NH}_{2}+\mathrm{H}$. Let us first construct the MOs correlation diagram for this process. The four crucial orbitals of $\mathbf{N H}_{3}$ are (a) the $\pi$ lone-pair orbital which correlates with the p- $\pi$ orbital of $\mathrm{NH}_{2}$ and (b) three $\sigma$ orbitals: the bonding $\left(\sigma_{\mathrm{NH}}\right)$ and antibonding $\left(\sigma_{\mathrm{NH}}^{*}\right)$ orbitals of the $\mathrm{NH}$ bond to be broken and the $3 \mathrm{~s}_{\mathrm{N}}$ Rydberg orbital on nitrogen. The $\sigma_{\mathrm{NH}}$ and $\sigma_{\mathrm{NH}}^{*}$ orbitals result from the combination of the in-plane $\mathrm{sp}_{2}$ hybrid of $\mathrm{NH}_{2}\left(\sigma_{\mathrm{N}}\right)$ and the $1 \mathrm{~s}$ orbital of $\mathrm{H}$. In the products $\sigma_{\mathrm{N}}$ lies below 1s; therefore $\sigma_{\mathrm{NH}}$ and $\sigma_{\mathrm{NH}}^{*}$ correlate with $\sigma_{\mathrm{N}}$ and $1 \mathrm{~s}$, respectively. The Rydberg orbital on nitrogen is not perturbed in the reaction. As shown in Figure $7\left(\right.$ a) $\sigma_{\mathrm{NH}}^{*}$ and $3 \mathrm{~s}_{\mathrm{N}}$ intend to cross but are forced to avoid each other since they both have $\sigma$ symmetry. This crossing is weakly avoided for the overlap between the strongly localized $\sigma_{\mathrm{NH}}^{*}$ orbital and the diffuse Rydberg orbital is small. Let us now consider the two excited configurations arising from the promotion of one $\pi$ electron to either $3 \mathrm{~s}_{\mathrm{N}}$ or $\sigma_{\mathrm{NH}}^{*}$. The lowest excited configuration $\left(\sigma_{\mathrm{NH}}^{2}, \pi, 3 \mathrm{~s}_{\mathrm{N}}\right)$ is destabilized when the hydrogen atom moves away and correlates with the high energy $\left(\sigma_{\mathrm{N}}^{2}, \pi, 3 \mathrm{~s}_{\mathrm{N}}\right)$ configuration of the products. At the same time the second configuration $\left(\sigma_{\mathrm{NH}}^{2}, \pi, \sigma_{\mathrm{NH}}^{*}\right)$ is monotonically stabilized and correlates with the $\left(\sigma_{\mathrm{N}}^{2}, \pi, 1 \mathrm{~s}_{\mathrm{H}}\right)$ configuration of the products. 


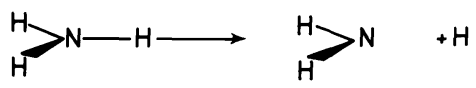

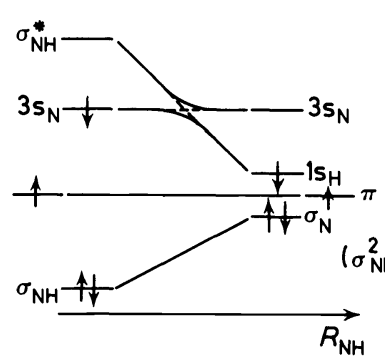

(a) Orbitals

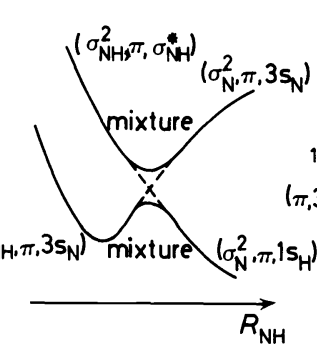

(b) Configurations

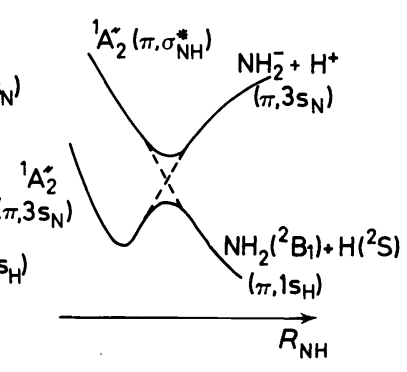

(c) States

Figure 7. Planar dissociation of ammonia.

These two configurations differ by the location of only one electron and, for this reason will be mixed by the monoelectronic part of the hamiltonian in the region where they are equienergetic. The MOs avoided crossing is then carried over at the one-electron configuration level [Figure 7(b)]. Of course, the states will also repel each other [Figure 7(c)], the gap between them being slightly greater than that between the avoiding configurations since the bielectronic terms (the difference between the instantaneous and averaged electron-electron repulsion) will add their contributions ${ }^{23,24}$. Physically a substantial electronic rearrangement is necessary for a hydrogen atom and $\mathrm{NH}_{2}\left({ }^{2} \mathrm{~B}_{1}\right)$ to form a planar $\left({ }^{1} \mathrm{~A}_{2}^{\prime \prime}\right) \mathrm{NH}_{3}$ molecule. The 1 s electron on the approaching $\mathrm{H}$ atom must be transferred to the Rydberg $3 \mathrm{~s}_{\mathrm{N}}$ orbital on $\mathrm{NH}_{2}$ before a bond may be initiated between the $\sigma_{\mathrm{N}}$ lone pair on the $\mathrm{N}$ atom and the $\mathrm{H}$ nucleus ${ }^{25}$.

To further illustrate these weakly avoided MOs crossings let us consider the photolytic dissociation of the benzylic $\mathrm{CH}$ bond in toluene. Only four MOs need be considered: (a) the highest occupied and lowest unoccupied $\pi$ and $\pi^{*}$ MOs which remain unperturbed during the reaction and (b) the bonding $\left(\sigma_{\mathrm{CH}}\right)$ and antibonding $\left(\sigma_{\mathrm{CH}}^{*}\right)$ MOs which correlate with the nonbonding orbitals of the benzyl radical $\left(\sigma_{\mathrm{C}}\right)$ and the hydrogen $(1 \mathrm{~s})$ respectively [Figure 8(a)]. If the plane of the ring is a plane of symmetry for the molecule and contains the $\mathrm{CH}$ bond to be broken the crossings between $\pi$ and $\sigma_{\mathrm{CH}}, \pi^{*}$ and $\sigma_{C H}^{*}$ are familiar symmetry allowed crossings. If not, these two crossings become weakly avoided and both correspond to a violent contraction process during which the delocalized $\pi$ and $\pi^{*}$ MOs of toluene become the strongly localized non-bonding $\sigma_{\mathrm{C}}$ and $1 \mathrm{~s}$ orbitals. Whether the plane of symmetry exists or not the $\pi \pi^{*}$ and $\sigma \sigma^{*}$ triplet states have the same symmetry and weakly avoid each other [Figure $8(\mathrm{~b})]$. The minimum in the ${ }^{3} \pi \pi^{*}$ state is about $83 \mathrm{kcal} / \mathrm{mole}$ above that of the ground state ${ }^{26}$. The energy required to break a $\mathrm{CH}$ bond is of the same order $(85 \mathrm{kcal} / \mathrm{mole})$ so that the overall process in the lowest triplet state would be isoenergetic. However, the ${ }^{3} \pi \pi^{*}$ minimum is separated from the ${ }^{3} \sigma \sigma^{*}$ diradical products by an energy barrier resulting from the weakly avoided crossing. When toluene is irradiated with 


$$
\mathrm{C}_{6} \mathrm{H}_{5} \mathrm{CH}_{3} \rightarrow \mathrm{C}_{6} \mathrm{H}_{5} \mathrm{CH}_{2}+\mathrm{H}
$$

(a) orbitals

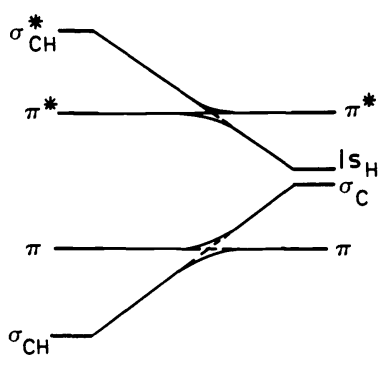

(b) states

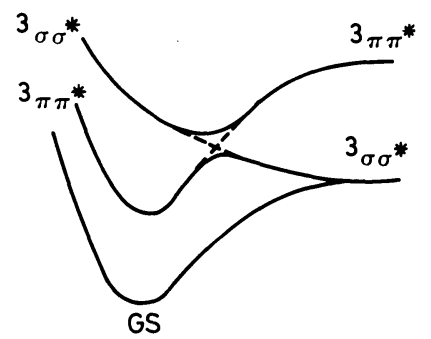

Figure 8. Photolytic dissociation of the benzylic $\mathrm{CH}$ bond of toluene.

light of sufficiently long wavelength the first photon excites the molecule in the ${ }^{3} \pi \pi^{*}$ state but the dissociation only proceeds when a second photon is absorbed. This additional photon brings the activation energy which is necessary to overcome the barrier in the lowest triplet energy surface ${ }^{27,28}$.

(3) Finally I would like to introduce a third situation in which the quasidegeneracy (but not the crossing) of MOs results in an avoided crossing between electronic states. These situations may be tentatively dubbed 'near-touching' MOs. An illustration of such cases may be found in the methane-plus-formaldehyde hydrogen abstraction reaction. Since we already know how the avoided character may be produced via nuclear skeleton distortions we might as well consider the simple symmetric system to elucidate the MO nature of the crossing. The MOs correlation diagram (Figure 9) displays two interesting features. First, there is a strongly avoided

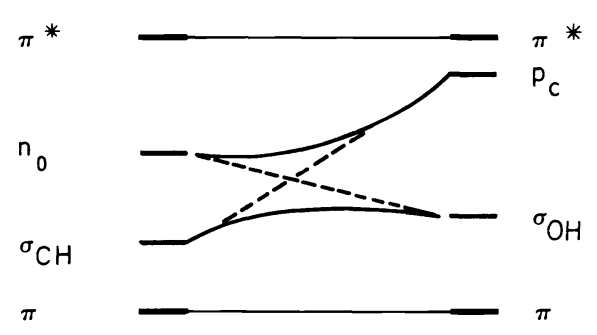

Figure 9. MOs correlation diagram in the formaldehyde-plus-methane hydrogen abstraction reaction.

crossing between the $\sigma_{\mathrm{CH}} \mathrm{MO}$ which is destabilized when the $\mathrm{CH}$ bond is elongated and the lone-pair orbital on the oxygen atom $n_{0}$ which is stabilized when the hydrogen approaches the oxygen atom to form the embryo of what will become the new $\mathrm{OH}$ bond. As a result of this avoided crossing $\sigma_{\mathrm{CH}}$ correlates with $\sigma_{\mathrm{OH}}$ and the $\mathrm{n}_{0}$ lone-pair correlates with the $\mathrm{sp}_{3}$ hybrid of 
the methyl group $\left(p_{c}\right)$. This crossing, though worthy of note, has no bearing on the problem at hand. The second important fact is that the non-bonding $\mathrm{p}_{\mathrm{c}}$ orbital is in the near vicinity of the $\pi^{*}$ orbital of the carbonyl group. In this typical situation three states may be expected: one diradical state where $\pi^{*}$ and $\mathrm{p}_{\mathrm{c}}$ each contain one electron and two zwitterionic states where $\pi^{*}$ or $\mathrm{p}_{\mathrm{c}}$ contains the pair of electrons. Usually the diradical is the most stable of the three states ${ }^{29,30}$. The ground state of the reactants correlates with one of the zwitterionic forms of the intermediates, the $n \pi^{*}$ state with the diradical hence the crossing of the two potential energy surfaces (Figures 10 and 4$)^{12}$. In sharp contrast with the two previous cases where the MOs

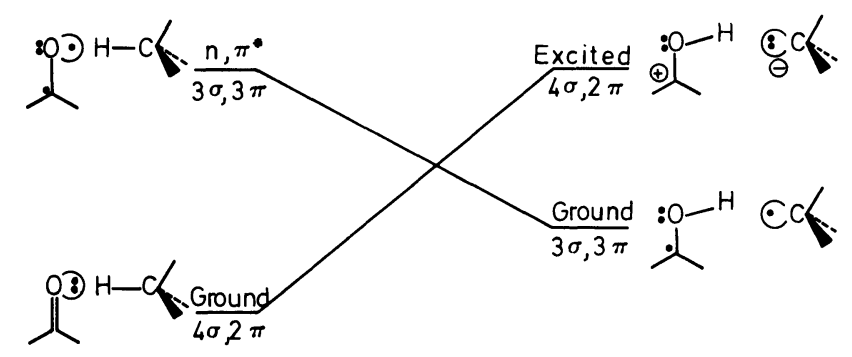

Figure 10. States correlation diagram in the formaldehyde-plus-methane hydrogen abstraction reaction.

were crossing (or weakly avoiding) each other roughly halfway along the reaction path the two important MOs here $\left(\pi^{*}\right.$ and $\left.p_{c}\right)$ do not cross but simply approach each other closely at the very end of the reaction, therefore permitting the facile jump of one electron from one of them to the other. This means that the energy that is necessary to promote one electron from $\mathrm{p}_{\mathrm{c}}$ to $\pi^{*}$ is smaller than the interelectronic repulsion of two electrons paired in $\mathrm{p}_{\mathrm{c}}$. Similar arguments are used in inorganic chemistry to explain why high-spin metal complexes are sometimes energetically preferred to low-spin complexes. The same phenomenon occurs in the sodium chloride case. When the internuclear distance increases the Coulombic attraction between $\mathrm{Cl}^{-}$and $\mathrm{Na}^{+}$decreases. At some point this energy is not sufficient to counteract the strong electron-electron repulsion of two electrons paired in one of the three chlorine $p$ orbitals. A more favourable situation is created when one of the chlorine electrons jumps to the $3 \mathrm{~s}$ orbital of sodium which is in a "near touching' situation with the chlorine lone-pair orbitals. The pair of radicals $(\mathrm{Cl} \cdot+\mathrm{Na} \cdot)$ thus created is the ground state of the elongated molecule.

\section{AVOIDED CROSSINGS AND MECHANISTIC PHOTOCHEMISTRY}

In mechanistic photochemistry avoided crossings are leakage channels along which the molecules flow from the higher down to the lower potential energy surfaces. In this final part we shall first show how avoided crossings may be experimentally created in cases where, at first sight, they seem improbable. Then we will illustrate the role of these crossings with (a) a 


\section{AVOIDED CROSSINGS IN PHOTOCHEMISTRY}

photochemical reaction: the disrotatory cyclization of butadiene, and (b) a photochemical process: the quenching of an excited species by a partner in its ground state.

\section{(1) Avoided crossings : the influence of substituents and medium}

The introduction of polar groups in a molecule or its immersion in a polar medium usually result in the lowering of the ionic electronic states. The cis-trans isomerization reaction of ethylene will serve to illustrate this point. The ground state wavefunction which, for the planar molecule, is an equal mixture of ionic and covalent terms, becomes purely covalent for the orthogonal $\left(90^{\circ}\right.$-twisted) geometry. The wavefunction of this orthogonal diradical $\mathrm{D}$ is

$$
\psi_{\mathrm{D}}=\phi_{\mathrm{A}}(1) \phi_{\mathrm{B}}(2)+\phi_{\mathrm{B}}(1) \phi_{\mathrm{A}}(2)
$$

The excited $\pi \pi^{*}$ singlet state on the other hand remains purely ionic throughout the entire twisting motion. The wavefunction of this zwitterion $\mathrm{Z}$ is the out-of-phase combination of the two possible polar structures

$$
\begin{aligned}
& \psi_{\mathrm{Z}}=\phi_{\mathrm{A}}(1) \phi_{\mathrm{A}}(2)-\phi_{\mathrm{B}}(1) \phi_{\mathrm{B}}(2) \\
& \frac{\ominus}{\mathrm{A}_{\mathrm{B}}} \oplus^{\prime} \mathrm{B}^{\prime} \cdot \Theta^{\prime}
\end{aligned}
$$

For a twist of $90^{\circ}, \mathrm{Z}$ is more than $100 \mathrm{kcal} /$ mole above $\mathrm{D}^{31}$ [Figure 11(a)]

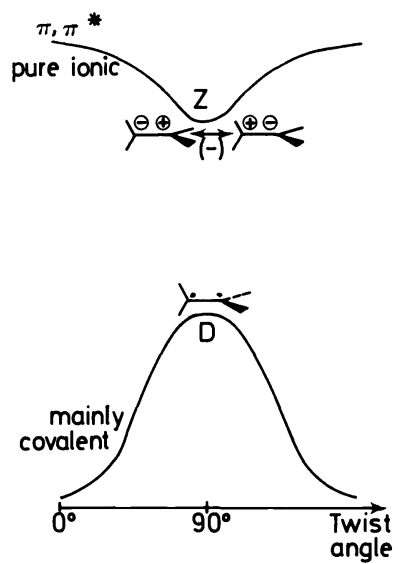

(a) ethylene

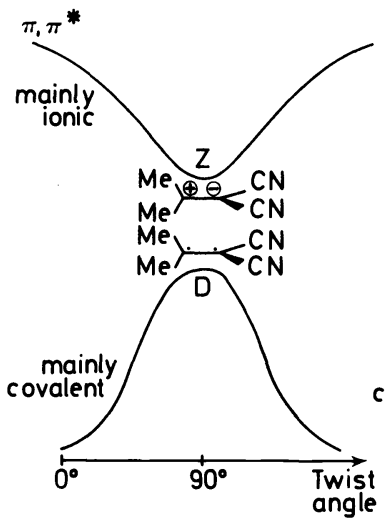

(b) polar olefin in non-polar solvent

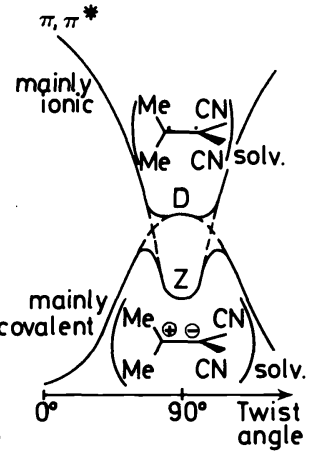

(c) polar olefin in polar solvent

Figure 11. Relative behaviour of the potential energy surfaces of the ground and $\left(\pi \pi^{*}\right)$ singlet states of ethylene.

but this rather large gap may be reduced:

(a) by the introduction into the molecule of polar groups such as cyano groups. The $\pi \pi^{*}$ singlet state of 1,1-dimethyl-2,2-dicyano-ethylene is significantly lowered [Figure 11(b)]. Its wavefunction has now acquired a small covalent character between 0 and $90^{\circ}$ but is still purely ionic at $90^{\circ}$ though no longer the combination of two equivalent polar forms. On the 
contrary the resonance structure in which the negative charge is stabilized by the neighbouring $C N$ groups $-\phi_{A}(1) \phi_{A}(2)$ if the cyano groups are un carbon $\mathrm{A}$-is now the major term in the wavefunction of $\mathrm{Z}$.

(b) by introducing the polar dicyano-olefin in a highly polar solvent. In this case $\mathrm{Z}$ might even become the ground state of the orthogonal form of ethylene. As a consequence the potential energy surfaces of the covalent ground state (which correlates with D) and the ionic $\pi \pi^{*}$ state (which correlates with Z) will cross if the mixing of the wavefunctions is forbidden but will avoid each other if this restriction is lifted [Figure 11(c)]. This situation is reminiscent of the sodium chloride case. The ground state of the system though remaining covalent during most of the twisting motion acquires more ionic character in the region of the potential energy barrier. Finally the secondary minimum in the ground state surface corresponds to a purely ionic wavefunction. Such a situation was predicted by L. Salem ${ }^{39}$ and has been confirmed by calculations made in our laboratory ${ }^{32}$. It is a beautiful example of how avoided crossings may be generated experimentally. The creation of a secondary minimum in the ground state and a double-minimum in the excited state are, from a practical point of view, consequences which look undoubtedly very promising.

\section{(2) Electrocyclic reactions}

The photochemically allowed disrotatory cyclization of butadiene in cyclobutene is characterized by two regions where singlet states potential energy surfaces cross or intend to cross. At the very beginning of the reaction the first crossing involves the antisymmetric $\left(\pi_{2} \rightarrow \pi_{3}\right)$ singly excited state $S_{1}\left({ }^{1} B_{2}\right)$ and a symmetric state $S_{2}\left({ }^{1} A_{1}\right)$. The nature of $S_{2}$ is fairly complex. At the one-electron configuration level the $\left(\pi_{2} \rightarrow \pi_{3}\right)^{2}$ doubly excited state is nearly degenerate with the $\left(\pi_{1} \rightarrow \pi_{3}\right)-\left(\pi_{2} \rightarrow \pi_{4}\right)$ singly excited state. Both states have the same symmetry $\left(\mathrm{A}_{1}\right)$ and strongly interact to give two linear combinations; one of them - the out-of-phase combination-ends up in a near-touching situation with $\mathrm{S}_{1}{ }^{33,34}$. Let us immediately remark that this near-touching situation of $S_{1}$ and $S_{2}$ certainly also occurs for longer conjugated polyenes. Now it is well known that in a photochemically allowed process the $S_{1}$ potential energy surface is rather flat since $S_{1}$ correlates with a singly excited state of the product. To be more precise this surface slowly rises when the reaction proceeds. This again is a general characteristic of the cyclization of polyenes since the gap between the HOMO and the LUMO of a polyene with $2 p \pi$ electrons is smaller than that of the $2(p-1) \pi$ electrons compound resulting from the closure. On the other hand the energy of $S_{2}$ decreases monotonically since this state correlates with the ground state of the product (this is also general for this type of reaction). The resulting crossing between $S_{1}$ and $S_{2}$ is allowed but will become weakly avoided if small distortions of the nuclear skeleton destroy the plane of symmetry of the system (geometry-dependent avoided crossing). The existence of this allowed crossing is probably a general property of a number of electrocyclic photochemical reactions. The second (avoided) crossing takes place roughly halfway along the reaction path and involves the symmetric ground-state $\mathrm{S}_{0}\left({ }^{1} \mathrm{~A}_{1}\right)$ of butadiene and the $\mathrm{S}_{2}$ 'doubly' excited state. This 


\section{AVOIDED CROSSINGS IN PHOTOCHEMISTRY}

crossing has already been analysed. It is model-dependent and results from an allowed crossing between MOs of different symmetry.

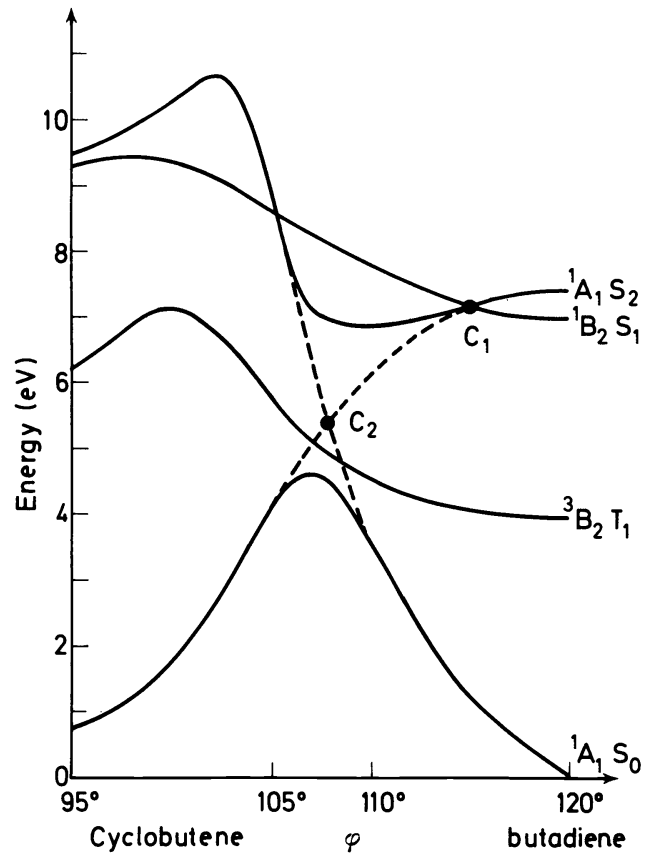

Figure 12. Calculated potential energy curves of the ground and low-lying excited states of butadiene during its disrotary cyclization in cyclobutene.

In Figure 12 we report the first extensive SCF.CI $(500 \times 500)$ calculations of the photochemical electrocyclic closure of butadiene ${ }^{35}$. The previously described crossings are labelled $\mathrm{C}_{1}$ and $\mathrm{C}_{2}$. Let us now examine the mechanistic consequences of these crossings. The first one $\left(C_{1}\right)$ is only $5 \mathrm{kcal} / \mathrm{mole}$ above the vertically excited $S_{1}$ state. This activation energy is small enough for the reaction to proceed in a time compatible with the lifetime of $S_{1}$. The crossing point $C_{1}$ will be easily reached and the reaction will be guided by the $S_{2}$ potential energy surface. The vertically excited ${ }^{1} B_{2}$ state is then a reservoir of excited species. $C_{1}$ is a valve which controls the flow of excited molecules toward the neighbouring well. The bottom of this well is below the vertically excited ${ }^{1} B_{2}$ state and is separated from the ground state maximum by an energy of $50 \mathrm{kcal} / \mathrm{mole}$. As we have noted, the gap must be rather large since the transition state is antiaromatic but the value we report here ${ }^{35}$ is overestimated. The ground state is fully correlated [via a configuration interaction (CI) which includes all the important doubly excited states] whereas the symmetric 'doubly' excited state $S_{2}$ is only partly so since our CI did not include the triple and quadruple excitations which would be necessary to reach a full correlation of this state. The well is a leakage channel toward the ground state surface. The detailed mechanism of the decay is not known but 


\section{ALAIN DEVAQUET}

certainly involves the coupling of the discrete vibational levels of the upper state with the quasi-continuum of the lower state. When the system has reached the ground state manifold it may either return to butadiene or produce cyclobutene.

The pattern of excited states we have described is probably more general. Let us then summarize its main features:

(i) the first excited singlet state $\mathrm{S}_{1}$-the reservoir of excited species-is slowly destabilized when the reaction proceeds;

(ii) the second excited singlet state $S_{2}$ is in a near-touching situation with $S_{1}$ and is stabilized;

(iii) the (allowed) crossing between $S_{1}$ and $S_{2}$ occurs at the very beginning of the reaction and allows the molecules which have some vibrational energy to populate the well which results from the second (avoided) crossing between $\mathrm{S}_{0}$ and $\mathrm{S}_{2}$;

(iv) this well brings the excited species in the vicinity of the maximum of the $S_{0}$ ground state surface. It is an exit channel from $S_{2}$ to $S_{0}$.

\section{(3) Quenching model ${ }^{36}$}

The intimate mechanism of the quenching of an excited molecule by a partner in its ground state is not clearly known at the present time. Yet a simple mechanism has been proposed for atom-atom and atom-diatomic molecule systems. For two reasons a schematic depiction of this mechanism will be briefly dealt with here. First it involves weakly avoided crossings and, second, it might well be generalized to explain certain types of molecular quenching processes.

(a) Atom-atom system

The general reaction occurring between an electronically excited atom $\mathrm{E}$ and a quenching atom $\mathrm{A}$ may be visualized as follows

$$
\mathrm{E}^{*}+\mathrm{A}+K_{\mathrm{i}} \rightarrow\left\{\mathrm{E}^{+}+\mathrm{A}^{-}\right\} \rightarrow \mathrm{E}+\mathrm{A}+K_{\mathrm{f}}
$$

where $K_{\mathrm{i}}$ and $K_{\mathrm{f}}$ represent the initial and final relative kinetic energies, respectively. For many quenching reactions the Coulomb attraction between $\mathrm{E}^{+}$and $\mathrm{A}^{-}$makes the ionic state energetically favourable relative to the initial state $\mathrm{E}^{*}+\mathrm{A}$ for a long range of internuclear distances. At shorter distances this state might even become more stable than the final state $\mathrm{E}+\mathrm{A}$. The relative position of the three potential energy curves is shown in Figure 13. Their two avoided crossings $(x$ and $y)$ are strongly dependent on the ionization potential of $\mathrm{E}$, the electronic affinity of $\mathrm{A}$ and the polarizability of the $\mathrm{E}^{+}+\mathrm{A}^{-}$intermediate. At $x$ the system switches from the initial state $\mathrm{E}^{*}+\mathrm{A}$ to the intermediate ionic state $\mathrm{E}^{+}+\mathrm{A}^{-}$, then, at $y$, from this ionic state to the final state $\mathrm{E}+\mathrm{A}$. The quantum-mechanical transition probabilities are of the Landau-Zener form and, as we have seen, depend upon the relative kinetic energy of the two fragments and the morphology of the diabatic potential energy curves at $x$ and $y$.

\section{(b) Atom-diatomic molecule system}

This simple model may be extended to the quenching of electronic excited 


\section{AVOIDED CROSSINGS IN PHOTOCHEMISTRY}

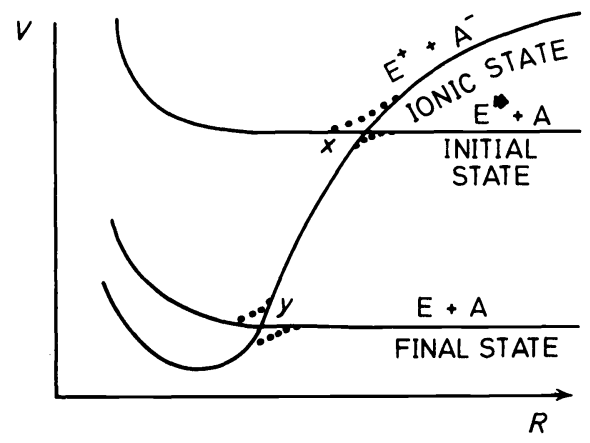

Figure 13. Potential energy curves in the quenching model for an atom-atom system.

atoms by molecules. If we consider the reaction

$$
\mathrm{Na}\left(3^{2} \mathrm{P}\right)+\mathrm{N}_{2}(V=0)+K_{\mathrm{i}} \rightarrow\left\{\mathrm{Na}^{+}+\mathrm{N}_{2}^{-}\left(V_{-}\right)\right\} \rightarrow \mathrm{Na}+\mathrm{N}_{2}\left(V_{\mathrm{f}}\right)+K_{\mathrm{f}}
$$

the ionic surface $\mathrm{Na}^{+}+\mathrm{N}_{2}^{-}$will be the convenient intermediate which facilitates the transition between the initial and final surfaces. It is true, however, that no $\mathrm{N}_{2}^{-}$ion is stable. Recent experiments on slow electron scattering have only revealed the existence of a metastable $\mathrm{N}_{2}^{-}$ion ${ }^{37}$. It is nevertheless

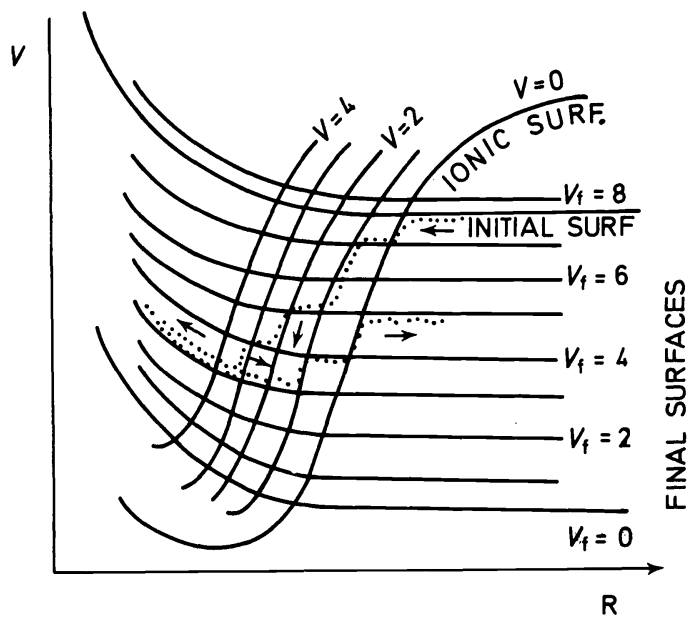

Figure 14. Potential energy curves in the quenching model for an atom-diatomic molecule system.

reasonable to assume that this metastable term is stabilized when a sufficiently strong Coulomb field (coming for example from the $\mathrm{Na}^{+}$ion) acts on the $\mathrm{N}_{2}^{-}$ion. The vibrational motion of the quenching molecule complicates the model. There is a sequence of both intermediate and final surfaces which are parametrized by electronic states as well as by vibrational quantum numbers ( $V_{-}$and $V_{\mathrm{f}}$ correspond to the vibrational levels of $\mathrm{N}_{2}^{-}$and $\mathrm{N}_{2}$ respectively) 
(Figure 14). Due to the multiple weakly avoided crossings, extremely complicated quenching paths must be considered. The problem is then the diffusion of the transition probability flux through the potential grid. Nevertheless two important physical conclusions may be established: (a) roughly half of the electronic excitation energy goes into vibration with the balance going into translation and/or rotation, and (b) the quenching is definitely non-resonant. In the case at hand the largest flux is predicted as going into $V_{\mathrm{f}}=5$ rather than to the near-resonant level $V_{\mathrm{f}}=7$.

To conclude let us recall that this model applies to the quenching of atoms having a weakly bound electron by a not strongly polar diatomic or small polyatomic molecule. These two conditions can undoubtedly be met by molecules of photochemical interest. The occurrence of ionic intermediates in photochemical reactions involving quenching processes would not be surprising and should be investigated.

\section{CONCLUSION}

To conclude this paper it is appropriate once more to emphasize the differences between the two types of electronic states which play-and will play in the future-the central role in the static and dynamic descriptions of avoided crossings ${ }^{38}$.

(1) The adiabatic states are simply the eigenstates of the electronic hamiltonian $H$. This means that they are purely electronic states (no vibrational part in their wavefunction) which diagonalize $H$. They obey the non-crossing rule and are perfect for describing the problems presented by a single permanent molecular state or, in other words, for static problems (for example, spectroscopic problems). The adiabatic potential energy curves are the corresponding eigenvalues of $H$ defined for each set of internuclear distances. They are the potential energies for the system if the atoms were not able to move. The physical nature of the adiabatic states changes along these curves. For example, in the sodium chloride case the lowest adiabatic state is purely ionic near the equilibrium distance, covalent and ionic in the avoided crossing region and finally purely covalent for very large internuclear separations.

(2) The diabatic states are also purely electronic states but are not eigenfunctions of the electronic hamiltonian. In addition they violate the noncrossing rule. They usually are physically simple wavefunctions: ionic or diradical valence-bond functions, well-defined simple one-electron MO configurations and the forces between them represent fairly well the forces between atoms moving with a small but finite velocity. These diabatic states are therefore appropriate for the two-state or many-state problems encountered in dynamics.

\section{ACKNOWLEDGEMENTS}

It is a pleasure to pay tribute here to Professor L. Salem. A significant part of my paper is based on his own work on the problem of avoided crossings ${ }^{39}$. The many concept-shaping discussions we have had were also very stimulating. 


\section{AVOIDED CROSSINGS IN PHOTOCHEMISTRY}

\section{REFERENCES}

1 J. von Neumann and E. P. Wigner, Z. Phys. 30, 467 (1929).

E. Teller, J. Phys. Chem. 41, 109 (1937).

K. Razi Naqvi and W. Byers-Brown, Internat. J. Quantum Chem. 6, 271 (1972).

K. Razi Naqvi, Chem. Phys. Letters, 15, 634 (1972).

R. B. Woodward and R. Hoffmann, J. Amer. Chem. Soc. 87, 395, 2046, 4388 (1965).

6 W. Th. A. M. van der Lugt and L. J. Oosterhoff, J. Amer. Chem. Soc. 91, 6042 (1969).

${ }^{7}$ L. Pauling and E. B. Wilson, Introduction to Quantum Mechanics, p 180. McGraw-Hill: New York (1935).

8 L. Landau, Phys. Z. Sowjet. 2,46 (1932).

9 C. Zener, Proc. Roy. Soc. A, 137, 696 (1932).

${ }^{10}$ E. E. Nikitin in Chemische Elementar Prozesse, p 43. Edited by H. Hartmann, Springer: Berlin (1968).

11 H. Eyring, J. Walter and G. Kimball, Quantum Chemistry, p 326. Wiley: New York (1965).

12 L. Salem, J. Amer. Chem. Soc. 96, 3486 (1974).

13 G. Herzberg, Diatomic Molecules, 2nd ed., p 372. Van Nostrand: New York (1950).

14 W. Kauzmann, Quantur Chemistry, p 536. Academic Press: New York (1957).

15 J. L. Magee, J. Chem. Phys. 8, 687 (1940).

16 R. Grice and D. R. Herschbach, Mol. Phys. 27, 159 (1974) and references therein.

17 H. C. Longuet-Higgins and G. W. Abrahamson, J. Amer. Chem. Soc. 87, 2045 (1965).

18 R. B. Woodward and R. Hoffmann, Angew. Chem., Internat. Ed. 8, 781 (1969).

19 M. Moffit, J. Chem. Phys. 22, 1820 (1954).

20 O. Sinanoğlu, J. Chem. Phys. 36, 706, 3198 (1962).

21 J. S. Wright and L. Salem, J. Amer. Chem. Soc. 94, 322 (1972).

22 A. E. Douglas, Disc. Faraday Soc. 35, 158 (1963).

23 J. A. Horsley and F. Flouquet, Chem. Phys. Letters, 5, 165 (1970).

24 J. A. Horsley and W. H. Fink, J. Chem. Phys. 50, 750 (1969).

25 G. Herzberg and H. C. Longuet-Higgins, Disc. Faraday Soc. 35, 77 (1963).

26 J. B. Birks, Photophysics of Aromatic Molecules, Wiley-Interscience: New York (1970).

${ }^{27}$ F. P. Schwartz and A. C. Albrecht, Chem. Phys. Letters, 9, 163 (1971).

28 J. Michl, Topics in Current Chemistry, 46, 1 (1974).

29 L. Salem and C. Rowland, Angew. Chem., Internat. Ed. 11, 92 (1972).

${ }^{30}$ L. Salem, Pure Appl. Chem. 33, 317 (1973).

31 U. Kaldor and I. Shavitt, J. Chem. Phys. 48, 191 (1968).

32 L. Salem and W. D. Stöhrer, Chem. Commun. 140 (1975).

33 T. H. Dunning, R. P. Hosteny and I. Shavitt, J. Amer. Chem. Soc. 95,5067 (1973).

${ }^{34}$ K. Schulten and M. Karplus, Chem. Phys. Letters, 14, 305 (1972).

35 D. Grimbert, A. Devaquet and G. Segal, J. Amer. Chem. Soc. 97 (1975).

${ }^{36}$ E. Bauer, E. R. Fisher and F. R. Gilmore, J. Chem. Phys. 51, 4173 (1969).

37 J. Schulz in Atomic Collision Processes, p 126. North Holland: Amsterdam (1964).

${ }^{38}$ T. F. O'Malley in Advances in Atomic and Molecular Physics, 7, 233 (1971).

39 L. Salem, C. Leforestier, G. Segal and R. Wetmore, J. Amer. Chem. Soc. 97,479 (1975). 